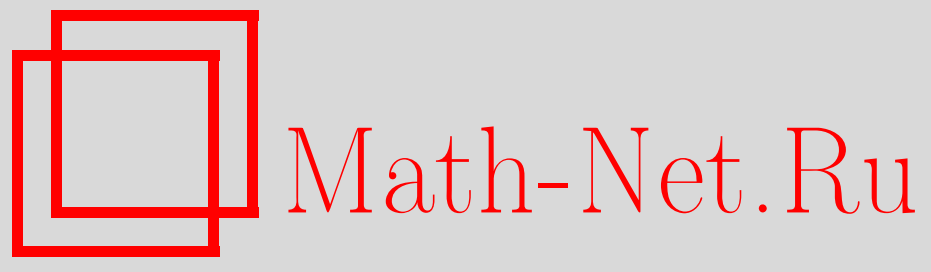

П. Г. Гриневич, С. П. Новиков, Спектрально мероморфные операторы и нелинейные системы, УМН, 2014, том 69, выпуск 5, 163-164

DOI: https://doi.org/10.4213/rm9621

Использование Общероссийского математического портала Math-Net.Ru подразумевает, что вы прочитали и согласны с пользовательским соглашением http://www . mathnet.ru/rus/agreement

Параметры загрузки:

IP : 54.198 .55 .26

26 апреля 2023 г., 06:45:11

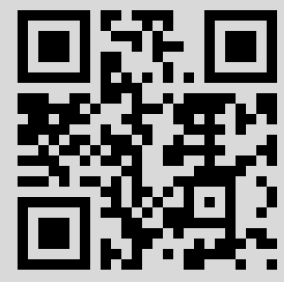




\section{Спектрально мероморфные операторы и нелинейные системы}

\section{П. Г. Гриневич, С. П. Новиков}

Мы рассматриваем ОД мероморфные линейные операторы, действующие на функции вещественной переменной $x \in \mathbb{R}$, вида $L=\partial_{x}^{n}+\sum_{n \geqslant i \geqslant 2} a_{n-i} \partial_{x}^{n-i}$ с коэффициентами, мероморфными около оси $\mathbb{R} \subset \mathbb{C}$. Мы скажем, что оператор является спектрально мероморфным, или s-мероморфным, около полюса $x_{j}$, если все решения уравнения $L \psi=\alpha \psi$ мероморфны около этой точки для всех $\alpha \in \mathbb{C}$. Мы будем изучать операторы, s-мероморфные около всех полюсов $x_{j} \in \mathbb{R}$.

Введем нужные функциональные пространства. Рассмотрим набор линейных подпространств $R_{j}$ вида $\sum_{-r_{j} \leqslant i<0} b_{i} y^{i}$ пространства всех лорановских полиномов. Определим пространство функций $F=F_{\left(\left\{x_{j} ; R_{j}\right\}\right)}$, мероморфных около оси $x \in \mathbb{R}$, с возможными полюсами в фиксированном дискретном множестве точек $x_{j} \in \mathbb{R}$ и разложениями Лорана по $y=x-x_{j}$ в фиксированном наборе линейных пространств $R_{j}$ для отрицательных степеней $y$. Положительные коэффициенты Лорана в полюсах $x_{j}$ должны удовлетворять только уравнениям, вытекающим из требования, что произведения функций из $F$ не имеют членов вида $y^{-1}$.

Лемма 1. Для любых финитных функиий $f(x), g(x), x \in \mathbb{R}$, из пространства $F$ скалярное произведение корректно определено формулой $\langle f, g\rangle=\int f(x) \bar{g}(\bar{x}) d x$, где интеграл берется по оси $x \in \mathbb{R}$ вне окрестности особенности и по контуру, обходящему особенность, около нее. Это скалярное произведение индефинитно, причем каждая особая точка $x_{j}$ дает (независимо от других $x_{k}$ ) $p_{j}$ отрицательных квадратов, где $p_{j}$ - размерность пространства $R_{j}$ отрицательных частей Лорана около $x_{j}$.

Доказательство легко следует из того, что, по определению пространства $F$, произведение функций не содержит степеней -1 около любого полюса. Риманова поверхность $\Gamma$ с отмеченной точкой $P$, локальным параметром $z=1 / \lambda$, дивизором $D$ степени $g$ (род) и мероморфной функцией $\alpha$ с полюсом только в $P$ порядка $k$ дает согласно конструкции BChK (Бурхнала-Чаунди-Кричевера [1]) ОД оператор $L \psi=\alpha \psi$ порядка $k$, алгебраический ранга 1 . Легко видеть, что этот оператор s-мероморфный. Он формально самосопряжен (симметричен), если выполнено условие $\mathbf{S A}:$ задана антиинволюиия $\tau: \Gamma \rightarrow \Gamma, \tau(P)=P$, такая, что $\tau(D)+D \sim K+2 P, \tau(\lambda)=-\bar{\lambda}$.

Особенности оператора $L$ определяют класс функций $F=F^{L}$ (см. выше). В периодическом случае мы строим пространство $F_{\kappa}^{L} \subset F^{L}$ из функций $f(x+T)=\kappa f(x)$, $|\kappa|=1$. В пространствах $F_{\kappa}^{L}$ число отрицательных квадратов конечно. Напомним, что дифференциалом квазиимпульса называется мероморфный дифференциал $d p$ с полюсом второго порядка только в точке $P, d p \sim d \lambda$, такой, что интегралы $\oint d p$ по всем циклам чисто вещественны. Тогда $\operatorname{Im} p=p_{I}$ выбирается как однозначная вещественная функция такая, что $\tau\left(p_{I}\right)=-p_{I}$, поскольку $\tau d p=\overline{d p}$. Уровень $p_{I}=0$ мы назовем каноническим контуром $C_{0}$.

Проекция $\alpha\left(C_{0}\right)$ контура $C_{0}$ на комплексную плоскость $\mathbb{C}$ совпадает со спектром оператора $L$ на всей прямой $x \in \mathbb{R}$ в пространстве $F^{L}$.

Теорема 2. Скалярное произведение в пространстве $F^{L}$ для алгебраического оператора L ранга 1, удовлетворяющего условию $\mathbf{S A}$, корректно определено и индефинитно. Оператор L самосопряжен относительно скалярного произведения (см. въше). Его спектр совпадает с множеством $\alpha\left(C_{0}\right)$, где $C_{0}-$ канонический контур. Скалярное произведение определяется интегралом по каноническому контуру $C_{0}$ со

Работа выполнена при поддержке РФФИ (грант № 14-01-00012).

DOI: $10.4213 / \mathrm{rm} 9621$ 
спектральной мерой $\Omega$. Если базис собственных функций на контуре $C_{0}$ полон в пространстве $F_{\kappa}^{L}$, то число отрицательных квадратов скалярного произведения в пространствах $F_{\kappa}^{L}$ определяется знаком спектральной меры $\Omega$ в соответствующих точках контура $C_{0}$ и является интегралом систем Гельфанда-Дикого $\dot{L}=[L, A]$.

Для доказательства теоремы рассмотрим функцию Бейкера-Ахиезера $\psi(\lambda, x) \sim$ $e^{\lambda x}$ (около $P$ ) с дивизором полюсов $D=\left(\gamma_{1}, \ldots, \gamma_{g}\right)$ и дуальную 1-форму $\psi^{*}(\mu, x) d \mu \sim$ $e^{-\mu x} d \mu$ (около $P$ ) с нулями $D=\left(\gamma_{1}, \ldots, \gamma_{g}\right)$. На языке скалярных функций БейкераАхиезера имеем $\psi^{*}(\mu, x) d \mu=\psi^{+}(\mu, x) \Omega$, где $\Omega=(1+o(1)) d \mu$ около $P$, с нулями $D+\tau(D), \tau(D)+D \sim K+2 P, K$ - канонический дивизор дифференциальных форм, $\Omega$ - спектральная мера на контур $C_{0}$. Мы имеем $L^{*} \psi^{*}=\mu \psi^{*}$, где $L^{*}$ сопряжен к $L$. Возможно, этот факт явно не отмечался в литературе. В работе [2] изучалось ядро типа Коши на $\Gamma: \omega(\lambda, \mu, x) \sim-d \mu /(\lambda-\mu)+O(1)$ около диагонали $\lambda=\mu$, $\omega \sim e^{\lambda x} o(1)$ при $\lambda \rightarrow P, \omega \rightarrow e^{-\mu x} o(1) d \mu$ при $\mu \rightarrow P$. Было найдено равенство $\partial_{x} \omega=-\psi(\lambda, x) \psi^{*}(\mu, x) d \mu$. Это равенство играет здесь ключевую роль. Следовательно, вычет по $x$ равен нулю для любого произведения собственных функций самосопряженного оператора $L$ около особенности. Тем самым, скалярное произведение (см. выше) корректно определено. При данном $\kappa$ число отрицательных квадратов является инвариантом римановой поверхности, которая сохраняется со временем: уравнение $e^{i p(z) t}=\kappa$ на контуре $C_{0}$ дает дискретный набор точек $z_{s} \in C_{0}$. Число точек, в которых величина $\left.d \Omega\right|_{z_{s}} \in \mathbb{R}$ отрицательна, конечно и дает число отрицательных квадратов скалярного произведения в пространстве $F_{\kappa}^{L}$. Полное пространство $F^{L}$ является прямым интегралом пространств $F_{\kappa}^{L}$.

ПримеР [3]. Для $k=2$ имеем $L=-\partial_{x}^{2}+u$. Пусть $u \sim r_{j}\left(r_{j}+1\right) /\left(x-x_{j}\right)^{2}+$ Reg. Для s-мероморфности регулярная часть потенциала обязана иметь в точке $x_{j}$ нулевые нечетные производные до порядка $2 r_{j}-1$ включительно. Все $R_{j}$ имеют вид $b_{1} y^{-r_{j}}+b_{2} y^{-r_{j}+2}+\cdots+b_{r_{j} / 2} y^{-2}$ - для четных $r_{j} \geqslant 2$ или $b_{1} y^{-r_{j}}+b_{2} y^{-r_{j}+2}+\cdots+$ $b_{\left(r_{j}+1\right) / 2} y^{-1}-$ для нечетных $r_{j} \geqslant 1$. Каждый полюс дает $\left[\left(r_{j}+1\right) / 2\right]$ отрицательных квадратов. Положительные члены лорановского разложения функций $f \in F$ в точках $x_{j}$ имеют нулевые коэффициенты при нечетных степенях $y^{2 i-1}, i \leqslant r_{j} / 2$, в первом случае и четных степенях $y^{2 i}, i \leqslant\left(r_{j}+1\right) / 2$, во втором случае. Уже для рода $g=1$ и вещественного эллиптического потенциала, отвечающего ромбической решетке, одна из конечных компонент спектра $\alpha\left(C_{0}\right)$ комплексна. Полнота собственных функций доказана в [3], так что число отрицательных квадратов сохраняется под действием $\mathrm{KdV}$ и высших KdV.

Предстоит доказать: 1. Если оператор s-мероморфный, то таков же и сопряженный оператор. 2. Все особенности $R_{j}$ s-мероморфных операторов реализуются алгебраическими. Их классификация - проблема. 3. Теорему полноты для симметричных алгебраических операторов.

\section{Список литературы}

[1] И. М. Кричевер, Функи. анализ и его прил., 11:1 (1977), 15-31. [2] P. G. Grinevich, A. Yu. Orlov, Problems of modern quantum field theory, Springer, Berlin, 1989, 86-106. [3] P. G. Grinevich, A. P. Novikov, Bull. Braz. Math. Soc. (N. S.), 44:4 (2013), 809-840.

П. Г. Гриневич (P. G. Grinevich)

ИТФ им. Л. Д. Ландау РАН

E-mail: pgg@landau.ac.ru

\section{С. П. Новиков (S. P. Novikov)}

University of Maryland, College Park, MD, USA;

Математический институт им. В. А. Стеклова РАН

E-mail: novikov@umd.edu
Представлено В. М. Бухштабером Принято редколлегией 02.09.2014 